(C) 2017 IEEE. Personal use of this material is permitted. Permission from IEEE must be obtained for all other uses, in any current or future media, including reprinting/republishing this material for advertising or promotional purposes, creating new collective works, for resale or redistribution to servers or lists, or reuse of any copyrighted component of this work in other works

This is the accepted manuscript of: Alessandro Mingotti ; Lorenzo Peretto ; Roberto Tinarelli ; Kenan Yiğit, "Simplified Approach to Evaluate the Combined Uncertainty in Measurement Instruments for Power Systems" IEEE Transactions on Instrumentation and Measurement ( Volume: 66 , Issue: 9, pp. 2258 - 2265, Sept. 2017 ) doi: 10.1109/TIM.2017.2677620 


\title{
Simplified Approach to Evaluate the Combined Uncertainty in Measurement Instruments for Power Systems
}

\author{
Alessandro Mingotti, Student Member, IEEE, Lorenzo Peretto, Senior Member, IEEE, \\ Roberto Tinarelli, Senior Member, IEEE, and Kenan Yiğit
}

\begin{abstract}
This paper presents the study of the propagation of the effects of uncertainties in a typical measurement system for modern power networks. Nowadays there is a strong demand from the electricity industry of simple and ready-to-use solutions for calculating uncertainty in such "new" measurement systems. To this purpose a simplified approach is presented whose results are shown to be in good agreement with those provided by conventional but more complex methods. Two applications, rather simple but very common, are considered in this paper. In the first one, the measurement of a voltage phasor is considered and the measurement chain is made by an low power instrument transformer (LPIT) and a converter used for adapting the level of signals from the LPIT to the inputs of intelligent electronic device. The second application deals with the measurement of active power of a system made by a low power voltage transformer (LPVT), a low power current transformer and a powermeter.
\end{abstract}

Index Terms-Accuracy class, low power current transformer (LPCT), low power voltage transformer (LPVT), smart grid, uncertainty.

\section{INTRODUCTION}

$\mathbf{M}$ ODERN power networks, also referred to as Smart Grids, feature new and nonconventional characteristics. They rely on powerful control architectures, communications infrastructures for real time management of the energy demand, bidirectional flow of energy (from prosumers to energy system operator and vice-versa), distributed generation, etc. The control and management of power apparatus and systems can be effectively performed if accurate information are provided by the measurement systems [made by sensing elements and Intelligent Electronic Device (IED)].

As far as the sensing elements are concerned, over the last years, nonconventional voltage and current transformers [low power instrument transformers (LPITs)] are rapidly replacing traditional potential and current transformers for many reasons: more compact in size and light; higher performance in

Manuscript received November 14, 2016; revised January 21, 2017; accepted February 20, 2017. Date of publication April 5, 2017; date of current version August 9, 2017. The Associate Editor coordinating the review process was Dr. Paolo Attilio Pegoraro.

A. Mingotti, L. Peretto, and R. Tinarelli are with the Dept. of Electrical, Electronic and Information Engineering, University of Bologna, 40126 Bologna, Italy (e-mail: roberto.tinarelli3@unibo.it).

$\mathrm{K}$. Yiğit is with the Dept. of Marine Engineering Operation, Yildiz Technical University, 34349 Istanbul, Turkey.

Color versions of one or more of the figures in this paper are available online at http://ieeexplore.ieee.org.

Digital Object Identifier 10.1109/TIM.2017.2677620 terms of accuracy, bandwidth, dynamic, safety; lower prices, etc. IEC TC38 "Instrument Transformers" has started recently to develop and issue a new Standard Series "61869" [1]-[4] with the intent to review and improve the Standards dealing with conventional voltage and current transformers but in particular to standardize the LPITs. When using such devices, it can occur that an item in-between the LPIT (usually referred to as Converter) and IED be required. The purpose is to adapt the signal levels and type (voltage, or current) between the two devices. For instance, it could be an impedance adapter for strengthen the LPIT signal in case of long distance cables (tens of meters) between LIPT and IED; or it could be an amplifier when the IED input requires "traditional amplitudes" for the voltage (100-110-200 V); or it could be a transconductance when the input if the IED must be a current $(1 / 5 \mathrm{~A})$. In the short term a new kind of converter is going to be used. It is referred to as Stand Alone Merging Unit (SAMU) [4]. A SAMU is a digital device, which collects all output signals from LPITs (both voltage and current instrument transformers), converts them into digital form and transmits the relevant sequence along one single carrier according to the IEC 61850 protocol [5]. A Standard, the IEC $61869-13$, is going to be published shortly dealing entirely with the SAMU characteristics as well as its errors.

\section{A. Scope of the Work}

The typical measurement system in modern power networks is made up of LPIT-Converter-IED. Aim of the study has been to come up with a simple and easy-to-use expression of uncertainty to be used in practical applications. In fact, as it is well known, often the operators, working in the design and setting-up of distribution substations, complain and get in trouble when they have to evaluate the overall uncertainty of such new measurement systems they design. They often do not have sufficient knowledge and skills to evaluate it. This is mainly because complex algorithms (as in the case of Guide to the expression of Uncertainty in Measurement (GUM) [6]) or complex procedures (as in the case of the Supplement 1 of GUM [7]), also for rather simple measurement systems as the ones considered here, must be implemented. For instance, in [8] and [9] uncertainty propagation in complex power systems is tackled by means of simulations featuring high computational burden and long run time. There is a strong demand from the electricity industry of simple 
and ready-to-use solutions for calculating uncertainty in such measurement systems.

Hence, starting from the original work [10], the intent of this paper is to provide suitable and usable expressions for all practitioners and system operators for evaluating "at a glance" the uncertainty affecting measurement results. Furthermore, this paper focuses on uncertainty affecting two important measured quantities: phasors and active powers at rated frequency. All this study, although it has been oriented to new measurement items nowadays used in power systems, it can be readily extended to measurement systems made by inductive instrument transformers.

This paper is divided into five sections. In Section II, an overview of methods for uncertainty evaluation is reported. In Section III, the proposed approach will be presented. Section IV deals with numerical examples, whereas, conclusions will be drawn in Section V.

\section{UNCERTAINTY EVALUATION}

As well known, the evaluation of the measurement uncertainty is a key point in any measurement process. According to [6] and [7], the measurement uncertainty relies on the assumption that the measurement result is a random variable, say $Y$, which is obtained by properly merging the random variables $X_{i}$ representing the input quantities upon which $Y$ depends through a model relating $Y$ to $X_{i}$. Random variables $X_{i}$ include the effects of uncertainty sources located in the measurement chain.

It is worthwhile noticing that, in this approach, random variables are used to model random as well as systematic effects. The latter occurs when a given "uncertainty" source turns into a constant effect on the measurement result but it is not possible to quantify its value. This "lack of knowledge" leads to the use of a random variable also in this case.

The approach in [6] and [7] looks like quite easy: the probability density function (pdf) associated with $Y$ is simply a proper combination of the pdfs associated with $X_{i}$. Of course, the way such pdfs should be combined depends on the measurement function, that is the above recalled model relating $Y$ to $X_{i}$. The drawback is that, except for the sum of two pdfs, which turns into their convolution, there are no mathematical tools for tackling operations between pdfs. To overcome this issue, two possible solutions are proposed by international Standards [6], [7].

In [6], the pdfs are represented by their means and standard deviations and a first-order Taylor series approximation is used to propagate them along the measurement function. Such an analytical approach provides exact results in a very limited number of situations and allows estimating the coverage probability merely if the resulting pdf is Normal. This occurs only when all the input pdfs are Normal or, according to central limit theorem, when several contributions must be considered.

The solution [7] relies on numerical simulations [Monte Carlo Method (MCM)]. Given that it is a statistically approach for estimating probability quantities the results are in this case always an approximation. However, if correctly implemented, the MCM features very accurate estimations and can

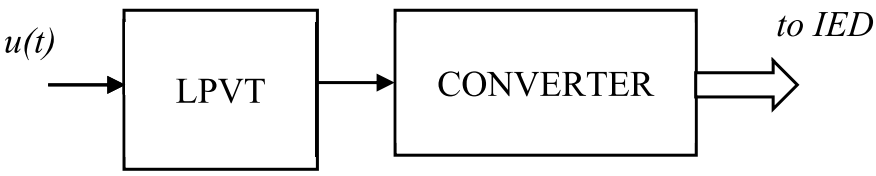

Fig. 1. Schematic of the considered measurement system.

be applied to a large class of problems. For these reasons, it is usually considered as a reference method for uncertainty evaluation and then used to validate any other proposal in the field of the measurement uncertainty propagation.

The main drawback of the above solutions is that their practical implementation is not straightforward because it may require to compute partial derivatives of a complex expression, according to [6] or to write and run several rows of software, according to [7] with often a high computational burden.

Different approaches have been proposed in the scientific literature to overcome some theoretical issues related to the use of [6], [7] (see [11]-[13]) but none of them has a practical implementation simpler than that of [6] or [7]. An interesting study which analyzes different methods for uncertainty evaluation can be found in [14].

\section{Proposed Simplified ApProACH}

In this section, two examples will be provided in order to show how expressions for uncertainty evaluation, according to the proposed approach, can be derived. Both examples deal with typical and significant medium voltage application and the hardware blocks involved in the study are according the scenario presented in Section I. The first example deals with the manufacturer issue to evaluate uncertainty in its products [low power voltage transformer (LPVT) + Converter]. On the contrary, the second example tackles the problem of uncertainty evaluation from the point of view of the end user, who buys the LPIT and the IED and wants to estimate uncertainty on the measured quantity.

\section{A. Uncertainty Due to the Combination of LPIT and Converter}

As stated in previous Sections, an important block part of a measurement equipment that is expected to be widely employed in the next future is the one depicted in Fig. 1. It is made by an LPVT and a Converter, which could be an impedance adapter, an amplifier, a transconductance or an SAMU.

According to [4], the accuracy performances of an LPVT are expressed by its accuracy class, which defines specific limits for the ratio error $\varepsilon_{\mathrm{VT}}$ and the phase error $\Delta \varphi_{\mathrm{VT}}$. The definitions of the above parameters are [4]

$$
\begin{gathered}
\varepsilon_{\mathrm{VT}} \triangleq \frac{k_{r} U_{s}-U_{p}}{U_{p}} \\
\Delta \varphi_{\mathrm{VT}} \triangleq \varphi_{s}-\varphi_{p} .
\end{gathered}
$$

In (1), $k_{r}$ is the rated transformation ratio, $U_{s}$ and $U_{p}$ are the rms values of the actual secondary and primary voltage, respectively, at rated frequency. In (2), $\varphi_{s}$ and $\varphi_{p}$ are the secondary and primary phase, respectively. 
Usually also the accuracy performances of the Converter will be expressed (according to what will be reported in the next 61869-13) in terms of ratio error $\varepsilon_{c}$ and phase error $\Delta \varphi_{s}$.

How can the whole system be characterized from a metrological point of view? According to the definitions reported in the new 61869 Standard series, the ratio error $\varepsilon$ and the phase error $\Delta \varphi$, that describe the difference between the input voltage $u(t)$ and the output voltage of the Converter, must be provided by the LPIT-Converter manufacturers. Of course, such parameters must be related to the rated value of $\varepsilon_{\mathrm{VT}}, \varepsilon_{c}$, $\Delta \varphi_{\mathrm{VT}}$ and $\Delta \varphi_{c}$, as expressed in the accuracy specifications of the LPVT and Converter. As a matter of fact, according to new Standard that IEC TC-38 is writing and soon publishing, all items in a power systems measurement chain (before the IED) must feature accuracy characteristics in terms of ratio errors and phase errors at rated frequency.

In this connection, let us denote by $\bar{U}_{\text {in }}$ the phasor of the input voltage $u(t)$, by $\bar{U}_{\mathrm{VT}}$ the phasor of the output voltage of the LPVT, by $\bar{U}_{\text {out }}$ the phasor of the Converter output. Moreover, let $k_{\mathrm{VT}}$ be the rated transformation ratio of the LPVT.

It is easy to show that

$$
\begin{aligned}
& \bar{U}_{\mathrm{out}}=\bar{U}_{\mathrm{VT}}\left(1+\varepsilon_{c}\right) e^{j \Delta \varphi_{c}} \\
& \bar{U}_{\mathrm{VT}}=\frac{\bar{U}_{\mathrm{in}}}{k_{\mathrm{VT}}}\left(1+\varepsilon_{\mathrm{VT}}\right) e^{j \Delta \varphi_{\mathrm{VT}}} .
\end{aligned}
$$

Hence

$$
\bar{U}_{\mathrm{out}}=\frac{\bar{U}_{\mathrm{in}}}{k_{\mathrm{VT}}}(1+\varepsilon \mathrm{VT}) e^{j \Delta \varphi_{\mathrm{VT}}}\left(1+\varepsilon_{c}\right) e^{j \Delta \varphi_{c}} .
$$

After some manipulations, (5) can be rewritten as

$$
\bar{U}_{\text {out }}=\frac{\bar{U}_{\text {in }}}{k_{\mathrm{VT}}}\left(1+\varepsilon \mathrm{VT}+q \varepsilon_{c}+\varepsilon \mathrm{VT} \varepsilon_{c}\right) e^{j\left(\Delta \varphi_{\mathrm{VT}}+\Delta \varphi_{c}\right)} .
$$

Therefore

$$
\begin{aligned}
\varepsilon & =\frac{k_{V T} U_{\text {out }}-U_{\text {in }}}{U_{\text {in }}}=\frac{U_{\text {in }}\left(1+\varepsilon_{\mathrm{VT}}+\varepsilon_{c}+\varepsilon_{\mathrm{VTT}} \varepsilon_{c}\right)-U_{\text {in }}}{U_{\text {in }}} \\
& =\varepsilon_{\mathrm{VT}}+\varepsilon_{c}+\varepsilon_{\mathrm{VT}} \varepsilon_{c} .
\end{aligned}
$$

Given that $\varepsilon_{\mathrm{VT}}$ and $\varepsilon_{c}$ are usually lower than $1 \%$, their product $\varepsilon_{\mathrm{VTT}} \varepsilon_{c}$ is at least 200 times lower than their sum $\varepsilon_{\mathrm{VT}}+\varepsilon_{c}$. Hence

$$
\varepsilon \cong \varepsilon_{\mathrm{VT}}+\varepsilon_{c} .
$$

As for the phase error $\Delta \varphi$, it is easy to verify that

$$
\Delta \varphi=\Delta \varphi_{\mathrm{VT}}+\Delta \varphi_{c} .
$$

According to [6], the terms at the right side of (8) and (9) are considered as random variables. Hence, also $\varepsilon$ and $\Delta \varphi$ are random variables whose pdfs depend on the pdfs associated with $\varepsilon_{\mathrm{VT}}, \varepsilon_{c}, \Delta \varphi_{\mathrm{VT}}$ and $\Delta \varphi_{c}$ given that, for the limited number of input quantities, the central limit theorem does not apply.

If, as usual for ratio and phase errors, no information, except their maximum values, is provided, both [6] and [7] suggest to assume uniform distributions (according to the maximum entropy principle) with zero mean and upper and lower limits given by the maximum values and minus the maximum values, respectively.

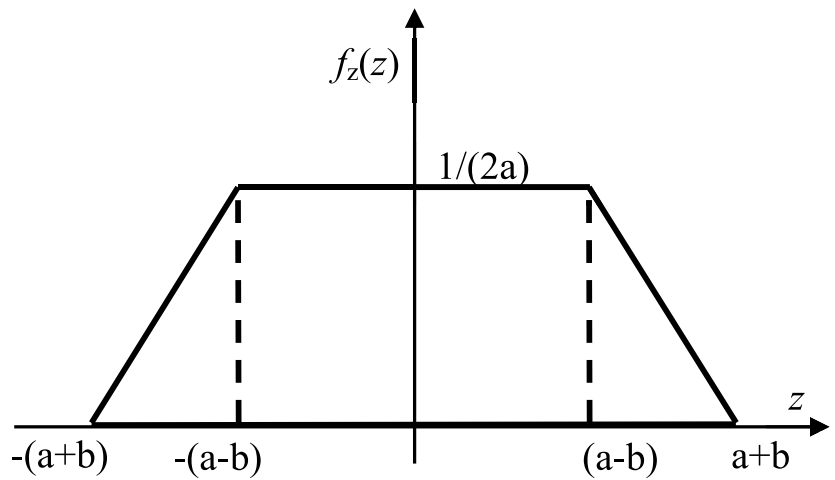

Fig. 2. Trapezoidal distribution obtained as convolution of two uniform distributions.

From a general point of view, the sum of two random variables turns into the convolution of their pdfs. It can be shown that under the above assumptions, the convolution provides a trapezoidal distribution. Let $x$ and $y$ be uniform random distributed variables ranging in $[-a ; a],[-b ; b]$, respectively, with density functions $f_{x}(x)$ and $f_{y}(y)$ such that

$$
\begin{aligned}
& f_{x}(x)= \begin{cases}\frac{1}{2 a}, & -a<x<a \\
0, & \text { otherwise }\end{cases} \\
& f_{y}(y)= \begin{cases}\frac{1}{2 b}, & -b<x<b \\
0, & \text { otherwise. }\end{cases}
\end{aligned}
$$

Their convolution $f_{z}(z)=f_{x}(x) * f_{y}(y)$ turns into:

$$
f_{Z}(z)=\int_{-\infty}^{+\infty} f_{X}(z-y) f_{Y}(y) d y=\int_{-b}^{+b} f_{X}(z-y) \frac{1}{2 b} d y .
$$

The integral (12) can be computed by dividing the $z$-axis into five different regions. By assuming $a>b$, the following results are obtained:

$$
f_{Z}(z)= \begin{cases}0, & z<-(a+b) \\ -\frac{a+b-z}{4 a b}, & -(a+b) \leq z<-(a-b) \\ \frac{1}{2 a}, & -(a-b) \leq z \leq a-b \\ \frac{a+b-z}{4 a b}, & a-b<z \leq a+b \\ 0, & a+b<z .\end{cases}
$$

Equation (13) clearly represents a trapezoidal distribution, as it is shown in Fig. 2.

Therefore, the pdfs of the ratio error $\varepsilon$ as well as of the phase error $\Delta \varphi$ are known and hence the coverage interval can be evaluated for any coverage probability. To this purpose, let $(-d,+d)$ be the searched $\gamma \%$-coverage interval, being $\gamma$ the coverage probability. The value of $d$ can be determined by solving the following equation:

$$
\int_{-d}^{+d} f_{z}(z) d z=\gamma
$$




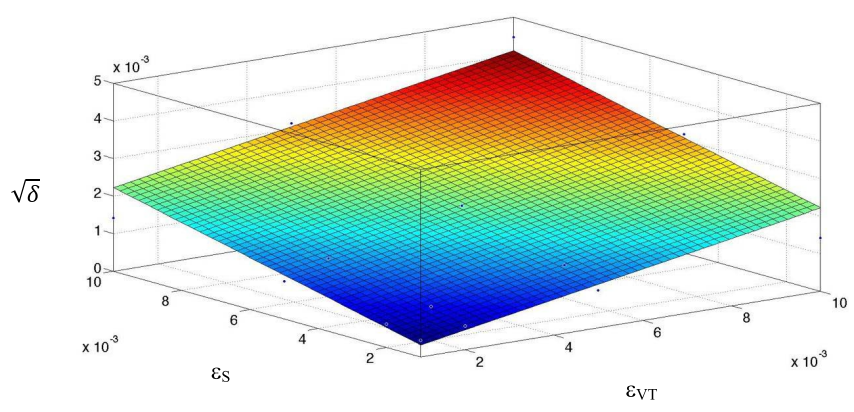

Fig. 3. Surface representing, for $\gamma=0.95$, the term $\sqrt{\delta}$ versus the ratio errors $\varepsilon_{\mathrm{VT}}$ and $\varepsilon_{c}$.

When $\gamma$ is high, it is reasonable to assume that it is $(a-b)<$ $d<(a+b)$. Then, (14) turns into this second-order equation

$$
\frac{1}{4 a b} d^{2}-\frac{a+b}{2 a b} d-\frac{3 b^{2}-a^{2}-2 a b}{4 a b}-\frac{a-b}{a}+\gamma=0
$$

whose solutions (as function of $\gamma \%$-coverage interval and by assuming $a$ and $b$ as constants) are

$$
d(\gamma)=a+b \pm \sqrt{4 b^{2}-4 a b\left(-\frac{a-b}{a}\right)}=a+b \pm \sqrt{\delta} .
$$

If $a=b$ the distribution turns into a triangular one, but (16) still holds because the contribution of the third equation in (13) becomes null. Of course, the positive root must be discarded given that it must be $(a-b)<d<(a+b)$. Hence

$$
d(\gamma)=a+b-\sqrt{\delta}
$$

Equations (16) and (17) provide the exact limits of the coverage interval for any coverage probability $\gamma$ and for given values of $a$ and $b$. However, even if their calculation is simpler and faster than directly applying one of the methods suggested in [6] and [7], its practical usage may be a bit unlikable.

In this connection, the radicand $\delta$ of (16) can be plotted, for a fixed value of $\gamma$, versus $a$ and $b$, thus providing a surface that can be approximated by a simpler equation. To this purpose, let us assume that $\gamma=0.95$. It must be observed that such a value is the typical value of coverage probability suggested by both [6] and [7] and that Standards [2]-[4] define standard values for both ratio and phase errors. Fig. 3 shows the surface relevant to $\sqrt{\delta}$ when the ratio errors $\varepsilon_{\mathrm{VT}}$ and $\varepsilon_{c}$ are considered. By applying a regression technique, it can be found that

$$
\sqrt{\delta} \cong 0.211\left(\varepsilon_{\mathrm{VT}}+\varepsilon_{c}\right)-10^{-4}
$$

it is a good approximation of the surface expression. Therefore, the value of the limit $d_{\varepsilon}$ of the $95 \%$-coverage interval of the ratio error $\varepsilon$ can be written

$$
d_{\varepsilon} \cong 0.789\left(\varepsilon_{\mathrm{VT}}+\varepsilon_{S}\right)+10^{-4} .
$$

Fig. 4 shows the surface relevant to $\sqrt{\delta}$ when the effects of phase errors $\Delta \varphi_{\mathrm{VT}}$ and $\Delta \varphi_{s}$ are taken into account. The application of regression technique provides

$$
\sqrt{\delta} \cong 0.08 \Delta \varphi_{c}+0.2 \Delta \varphi_{\mathrm{VT}}+17.2 \Delta \varphi_{c} \Delta \varphi_{\mathrm{VT}}-5.9 \Delta \varphi_{\mathrm{VT}}{ }^{2}
$$

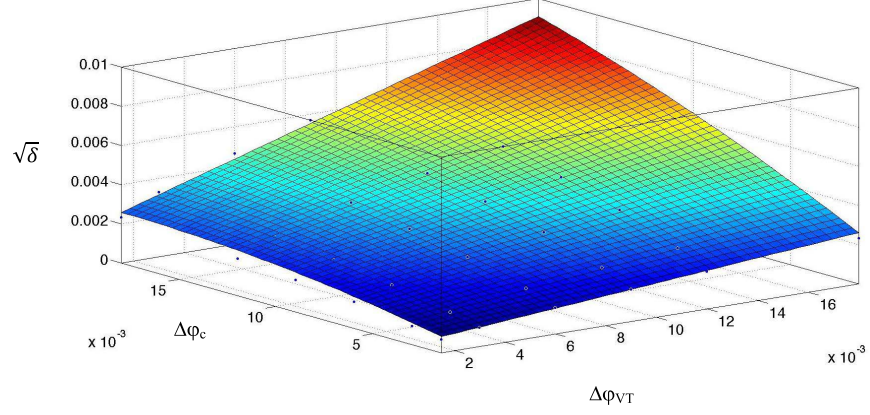

Fig. 4. Surface representing, for $\gamma=0.95$, the term $\sqrt{\delta}$ versus the phase errors $\Delta \phi_{\mathrm{VT}}$ and $\Delta \phi_{\mathrm{c}}$

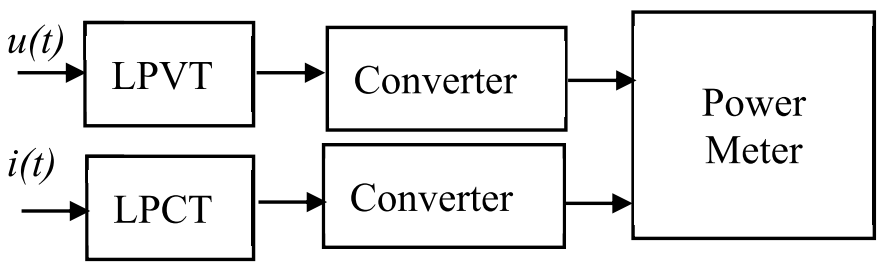

Fig. 5. Schematic representation of Powermeter and relevant voltage and current transformers.

which leads to the following expression of the limit $d_{\Delta \varphi}$ of the $95 \%$-coverage interval relevant to the phase error $\Delta \varphi$ :

$d_{\Delta \varphi} \cong 0.92 \varphi_{c}+0.8 \Delta \varphi_{\mathrm{VT}}-17.2 \Delta \varphi_{c} \Delta \varphi_{\mathrm{VT}}+5.9 \Delta \varphi_{\mathrm{VT}}{ }^{2}$. (21)

In the next section, a numerical validation of the above expressions will be provided.

\section{B. Powermeter}

In this second example the propagation of uncertainty in case of two measurement lines made by LPIT and Converter through a measurement function is provided to show how the end-user can take advantage of the proposed simplified approach. Active power has been chosen as measurement function as it represents, along with the phasors of voltage and current, the most widely measured quantity among electric system operators. The typical block diagram of a modern Powermeter, made by the IED, by the LPVT, low power current transformer (LPCT) and relevant converters, is shown in Fig. 5.

For the sake of simplicity but without loss of generality let's assume that the accuracy performance of the LPVT + Converter are still defined in (1) and (2). As for the LPCT + Converter, the Standard [2] defines the accuracy class in term of the ratio error $\varepsilon_{\mathrm{CT}}$ and the phase error $\Delta \varphi_{\mathrm{CT}}$. Also for this device let's consider such values as for the whole system LPCT + Converter. The definitions of the latter parameters are [2]

$$
\begin{aligned}
\varepsilon_{\mathrm{CT}} & \triangleq \frac{k_{r} U_{s}-I_{p}}{I_{p}} \\
\Delta \varphi_{\mathrm{CT}} & \triangleq \varphi_{s}-\varphi_{p} .
\end{aligned}
$$

In (22), $k_{r}$ is the rated transformation ratio, $U_{s}$ is the rms value of the actual secondary voltage (the output of an LPCT is a voltage), $I_{p}$ is the rms value of the actual primary current. $U_{s}$ and $I_{p}$ are evaluated at rated frequency. In (23), $\varphi_{s}$ and $\varphi_{v}$ are the secondary and primary phase, respectively. 
As for the Powermeter, it is usually characterized by a gain error $g$.

The metrological characterization of the whole measurement system can be done by evaluating the relative difference $\Delta(P /(P))$ between the input power $P$ and the power $P_{m}$ that results by adding to $P$ the unknown errors due to the LPVT, LPCT, and by the Powermeter.

In this connection, let us denote by $\bar{U}_{\text {in }}$ and $\bar{I}_{\text {in }}$ the phasors of the input voltage $u(t)$ and current $i(t)$, respectively, by $k_{\mathrm{VT}}$ and $k_{\mathrm{CT}}$ the rated transformation ratio of the LPVT and LPCT, respectively, and by $\phi$ the angle between $\bar{U}_{\text {in }}$ and $\bar{I}_{\text {in }}$.

By defining

$$
\begin{aligned}
\bar{U}_{\mathrm{out}} & =\frac{U_{\mathrm{in}}}{k_{\mathrm{VT}}}\left(1+\varepsilon_{V}\right) e^{j \Delta \varphi_{\mathrm{VT}}} \\
\bar{U}_{i-\text { out }} & =\frac{I_{\mathrm{in}}}{k_{\mathrm{CT}}}\left(1+\varepsilon_{C}\right) e^{j\left(\varphi+\Delta \varphi_{\mathrm{CT}}\right)}
\end{aligned}
$$

the output voltages of the LPVT and LPCT, respectively, (for the LPCT the quantity $k_{\mathrm{CT}}$ has the dimension of a conductance), then the above defined active power $P_{m}$ can be written as

$$
P_{m}=\operatorname{Re}\left(\bar{U}_{\text {out }} \bar{U}_{i-\text { out }}^{*}\right)+g * \operatorname{Re}\left(\bar{U}_{\text {out }} \bar{U}_{i-\text { out }}^{*}\right)
$$

which turns into

$$
\begin{aligned}
P_{m}= & \frac{U_{\text {in }}}{k_{\mathrm{VT}}}\left(1+\varepsilon_{\mathrm{VT}}\right) \frac{I_{\text {in }}}{k_{\mathrm{CT}}}\left(1+\varepsilon_{\mathrm{CT}}\right) \cos \left(\Delta \varphi_{\mathrm{VT}}-\Delta \varphi_{\mathrm{CT}}-\varphi\right) \\
& +g \frac{U_{\mathrm{in}}}{k_{\mathrm{VT}}}\left(1+\varepsilon_{\mathrm{VT}}\right) \frac{I_{\text {in }}}{k_{\mathrm{CT}}}\left(1+\varepsilon_{\mathrm{CT}}\right) \\
& \times \cos \left(\Delta \varphi_{\mathrm{VT}}-\Delta \varphi_{\mathrm{CT}}-\varphi\right) .
\end{aligned}
$$

A real function $f(x)$ that is infinitely differentiable at a given real number $x_{0}$ can be expressed as Taylor series

$f_{(x)}=f_{\left(x_{0}\right)}+{f^{\prime}\left(x_{0}\right)}\left(x-x_{0}\right)+\frac{f^{\prime \prime}\left(x_{0}\right)}{2 !}\left(x-x_{0}\right)^{2}+\cdots$.

Therefore, it is

$$
\begin{aligned}
\cos \left(\Delta \varphi_{\mathrm{VT}}-\Delta \varphi_{\mathrm{CT}}-\varphi\right) & \cong \cos (-\varphi)+\sin \left(\Delta \varphi_{\mathrm{VT}}-\Delta \varphi_{\mathrm{CT}}\right) \\
& =\cos (\varphi)+\sin \left(\Delta \varphi_{\mathrm{VT}}-\Delta \varphi_{\mathrm{CT}}\right)
\end{aligned}
$$

being $\cos (-\varphi)=\cos (\varphi)$. On the basis of (27) and (29) turns into

$$
\begin{aligned}
P_{m} \cong & \frac{U_{\mathrm{in}} I_{\mathrm{in}}}{k_{\mathrm{VT}} k_{\mathrm{CT}}}\left(1+\varepsilon_{\mathrm{VT}}\right)\left(1+\varepsilon_{\mathrm{CT}}\right) \\
& \times\left[\cos \varphi-\sin \varphi\left(\Delta \varphi_{\mathrm{VT}}-\Delta \varphi_{\mathrm{CT}}\right)\right] \\
& +g \frac{U_{\mathrm{in}} I_{\mathrm{in}}}{k_{\mathrm{VT}} k_{\mathrm{CT}}}\left(1+\varepsilon_{\mathrm{VT}}\right)\left(1+\varepsilon_{\mathrm{CT}}\right) \\
& \times\left[\cos \varphi-\sin \varphi\left(\Delta \varphi_{\mathrm{VT}}-\Delta \varphi_{\mathrm{CT}}\right)\right] .
\end{aligned}
$$

After some manipulations, by neglecting the terms that are at least one order of magnitude lower than the others and by putting

$$
P=\frac{U_{\text {in }} I_{\text {in }}}{k_{\mathrm{VT}} k_{\mathrm{CT}}} \cos \varphi
$$

Equation (30) can be rewritten as

$$
\begin{aligned}
P_{m} \cong & P-\frac{U_{\mathrm{in}} I_{\text {in }}}{k_{\mathrm{VT}} k_{\mathrm{CT}}} \sin \varphi\left(\Delta \varphi_{\mathrm{VT}}-\Delta \varphi_{\mathrm{CT}}\right) \\
& +\varepsilon_{\mathrm{VT}} P+\varepsilon_{\mathrm{CT}} P+g P .
\end{aligned}
$$

Therefore, it is

$$
\begin{aligned}
\frac{\Delta P}{P}= & \frac{P_{m}-P}{P} \cong \Delta \varphi_{\mathrm{CT}} \tan \varphi-\Delta \varphi_{\mathrm{VT}} \tan \varphi \\
& +\varepsilon \mathrm{VT}+\varepsilon_{\mathrm{CT}}+g .
\end{aligned}
$$

According to [6], the terms at the right side of (33) must be considered as random variables. Hence, also $(\Delta P /(P))$ is a random variable whose pdf depends on the pdfs associated with $\varepsilon_{\mathrm{VT}}, \varepsilon_{\mathrm{CT}}, \Delta \varphi_{\mathrm{VT}}, \Delta \varphi_{\mathrm{CT}}$, and $g$. However, given that $(\Delta P /(P))$ is a linear combination of random variables, its variance $\sigma_{(\Delta P /(P))^{2}}$ is provided, in accordance with [15], by

$$
\sigma_{\frac{\Delta P}{P}}^{2}=\sigma_{\Delta \varphi_{\mathrm{CT}}}^{2}+\sigma_{\Delta \varphi_{\mathrm{VT}}}^{2}+\sigma_{\varepsilon_{\mathrm{CT}}}^{2}+\sigma_{\varepsilon_{\mathrm{VT}}}^{2}+\sigma_{g}^{2}
$$

where $\sigma_{\Delta \varphi_{\mathrm{CT}}}^{2}, \sigma_{\Delta \varphi_{\mathrm{VT}}}^{2}, \sigma_{\varepsilon_{\mathrm{CT}}}^{2}, \sigma_{\varepsilon_{\mathrm{VT}}}^{2}, \sigma_{g}^{2}$ are the variances of the random variables $\Delta \varphi_{\mathrm{CT}} \tan \varphi, \Delta \varphi_{\mathrm{VT}} \tan \varphi, \varepsilon_{\mathrm{VT}}, \varepsilon_{\mathrm{CT}}, \mathrm{g}$, respectively.

If the pdf of $(\Delta P /(P))$ is assumed to be Normal, (34) allows to easily determine the limit $d_{(\Delta P /(P))}$ of any $\gamma \%$-coverage interval $\left(-d_{(\Delta P /(P))}, d_{(\Delta P /(P))}\right)$

$$
d_{\frac{\Delta P}{P}}=k \sigma_{\frac{\Delta P}{P}}
$$

being $k$ a proper coverage factor.

The combination of (34) and (35) is a very simple way to estimate the relative uncertainty of the power measured with the system in Fig. 5.

Unfortunately, if the pdfs of the random variables which $(\Delta P /(P))$ depends on are, as it is usual, uniforms, the application of typical statistical tests such as chi-square, KolmogorovSmirnov and Lilliefors leads to reject the hypothesis of Normal distribution of $(\Delta P /(P))$. Under these conditions, $(35)$ is still a very simple expression but provides an approximated result.

In the following section, a numerical validation of the above expressions will be provided.

\section{NUMERICAL EXAMPLES}

\section{A. Uncertainty Due to the Combination of LPIT and Converter}

The correctness of the approximation done in (8) and the accuracy of the approximated expressions (19) and (21) for the evaluation of the limits $d_{\varepsilon}$ and $d_{\Delta \varphi}$ of the $95 \%$-coverage interval of the ratio and phase errors $\varepsilon$ and $\Delta \varphi$ have been evaluated by comparing the results provided by (17), (19), and (21) with those provided by an MCM with 100000 iterations.

As far as $d_{\varepsilon}$ is concerned, all the possible combinations of values of the ratio errors $\varepsilon_{\mathrm{VT}}$ and $\varepsilon_{s}$ as defined in [3] and [4] have been considered. If, for the sake of simplicity, it is assumed that all the LPIT are working close to their full scale, $\varepsilon_{\mathrm{VT}}$ and $\varepsilon_{s}$ can be $0.1 \%, 0.2 \%, 0.5 \%$, and $1 \%$. If different working condition are considered, different ratio errors values must be taken into account in accordance with [3] and [4]. Tables I-III show the values of $d_{\varepsilon}$ obtained by 
TABLE I

LiMiT $d_{\varepsilon}$ (IN \%) COMPUTED BY APPLYING AN MCM PROCEDURE

\begin{tabular}{|c|c|c|c|c|}
\hline $\boldsymbol{\varepsilon}_{\mathbf{s}, \boldsymbol{\varepsilon}_{\text {VT }}}$ & $\mathbf{0 . 1}$ & $\mathbf{0 . 2}$ & $\mathbf{0 . 5}$ & $\mathbf{1}$ \\
\hline $\mathbf{0 . 1}$ & 0.15 & 0.24 & 0.50 & 0.96 \\
\hline $\mathbf{0 . 2}$ & 0.24 & 0.31 & 0.56 & 1.00 \\
\hline $\mathbf{0 . 5}$ & 0.50 & 0.56 & 0.77 & 1.18 \\
\hline $\mathbf{1}$ & 0.96 & 1.00 & 1.18 & 1.55 \\
\hline
\end{tabular}

TABLE II

LIMIT $d_{\varepsilon}($ IN \%) COMPUTED BY APPLYING (17)

\begin{tabular}{|c|c|c|c|c|}
\hline $\boldsymbol{\varepsilon}_{\mathrm{s}, \boldsymbol{\varepsilon}_{\mathbf{V T}}}$ & $\mathbf{0 . 1}$ & $\mathbf{0 . 2}$ & $\mathbf{0 . 5}$ & $\mathbf{1}$ \\
\hline $\mathbf{0 . 1}$ & 0.16 & 0.24 & 0.50 & 0.96 \\
\hline $\mathbf{0 . 2}$ & 0.24 & 0.31 & 0.56 & 1.00 \\
\hline $\mathbf{0 . 5}$ & 0.50 & 0.56 & 0.78 & 1.18 \\
\hline $\mathbf{1}$ & 0.96 & 1.00 & 1.18 & 1.55 \\
\hline
\end{tabular}

TABLE III

LIMIT $d_{\varepsilon}($ IN \%) COMPUTED BY APPLYING (19)

\begin{tabular}{|c|c|c|c|c|}
\hline $\boldsymbol{\varepsilon}_{\mathrm{s}, \boldsymbol{\varepsilon}_{\mathbf{V T}}}$ & $\mathbf{0 . 1}$ & $\mathbf{0 . 2}$ & $\mathbf{0 . 5}$ & $\mathbf{1}$ \\
\hline $\mathbf{0 . 1}$ & 0.17 & 0.25 & 0.48 & 0.88 \\
\hline $\mathbf{0 . 2}$ & 0.25 & 0.33 & 0.56 & 0.96 \\
\hline $\mathbf{0 . 5}$ & 0.48 & 0.56 & 0.80 & 1.19 \\
\hline $\mathbf{1}$ & 0.88 & 0.96 & 1.19 & 1.59 \\
\hline
\end{tabular}

TABLE IV

Limit $d_{\Delta \varphi}$ (IN CRAD) COMPUTED By APPLYING AN MCM PROCEDURE

\begin{tabular}{|l|c|c|c|c|c|c|}
\hline$\Delta \boldsymbol{\varphi}_{\text {s, }}$ & & & & & & \\
$\Delta \boldsymbol{\varphi}_{\mathbf{V T}}$ & $\mathbf{0 . 1 5}$ & $\mathbf{0 . 3}$ & $\mathbf{0 . 6}$ & $\mathbf{0 . 9}$ & $\mathbf{1 . 2}$ & $\mathbf{1 . 8}$ \\
\hline $\mathbf{0 . 1 5}$ & 0.23 & 0.36 & 0.62 & 0.88 & 1.16 & 1.72 \\
\hline $\mathbf{0 . 3}$ & 0.35 & 0.47 & 0.71 & 0.97 & 1.23 & 1.77 \\
\hline $\mathbf{0 . 6}$ & 0.62 & 0.71 & 0.93 & 1.17 & 1.42 & 1.94 \\
\hline $\mathbf{0 . 9}$ & 0.89 & 0.97 & 1.17 & 1.39 & 1.64 & 2.12 \\
\hline $\mathbf{1 . 2}$ & 1.16 & 1.23 & 1.42 & 1.63 & 1.88 & 2.34 \\
\hline $\mathbf{1 . 8}$ & 1.71 & 1.78 & 1.94 & 2.13 & 2.34 & 2.80 \\
\hline
\end{tabular}

applying the reference method (MCM, Table I), formula (17) obtained by discarding the product $\varepsilon_{\mathrm{VTT}} \varepsilon_{s}$ (Table II) and the approximation (19) (Table III).

The first observation arises by comparing Tables I and II. The limits of the confidence intervals shown in that tables are always equal except in two situations where they differ of $0.01 \%$. This confirms that the contribution of the product $\varepsilon \mathrm{VT} \varepsilon_{s}$ can be assumed as negligible. From the comparison of Table III with Table I (or Table II) it can be learned that the error resulting in the application of the proposed approximated formula (19) is very low, being it always not greater than $0.04 \%$ except when the ratio errors largely differ one from each other. In such a case, the simplified approach tends to become useless because the distribution associated with the larger ratio error prevails. For instance, it is the case when the two ratio errors are $1 \%$ and $0.1 \%$. In conclusion, according to standard accuracy classes, the proposed approach is not recommended only when ratio errors of $1 \%$ and $0.1 \%$ are considered.

As for $d_{\Delta \varphi}$, all the possible combination of values of the phase errors $\Delta \varphi_{\mathrm{VT}}$ and $\Delta \varphi_{s}$ as defined by [3] and [4] have been considered. Hence, each of them can be $0.15,0.3,0.6$, $0.9,1.2$, and $1.8 \mathrm{crad}$. Tables IV-VI show the values of $d_{\Delta \varphi}$
TABLE V

LIMIT $d_{\Delta \varphi}$ (IN CRAD) COMPUTED BY APPLYING (17)

\begin{tabular}{|c|c|c|c|c|c|c|}
\hline $\begin{array}{l}\Delta \boldsymbol{\varphi}_{\text {s, }} \\
\Delta \boldsymbol{\varphi}_{\text {VT }}\end{array}$ & $\mathbf{0 . 1 5}$ & $\mathbf{0 . 3}$ & $\mathbf{0 . 6}$ & $\mathbf{0 . 9}$ & $\mathbf{1 . 2}$ & $\mathbf{1 . 8}$ \\
\hline $\mathbf{0 . 1 5}$ & 0.23 & 0.36 & 0.62 & 0.89 & 1.16 & 1.72 \\
\hline $\mathbf{0 . 3}$ & 0.36 & 0.47 & 0.71 & 0.97 & 1.23 & 1.77 \\
\hline $\mathbf{0 . 6}$ & 0.62 & 0.71 & 0.93 & 1.17 & 1.42 & 1.94 \\
\hline $\mathbf{0 . 9}$ & 0.89 & 0.97 & 1.17 & 1.40 & 1.64 & 2.13 \\
\hline $\mathbf{1 . 2}$ & 1.16 & 1.23 & 1.42 & 1.64 & 1.86 & 2.34 \\
\hline $\mathbf{1 . 8}$ & 1.72 & 1.77 & 1.94 & 2.13 & 2.34 & 2.80 \\
\hline
\end{tabular}

TABLE VI

LIMIT $d_{\Delta \varphi}$ (IN CRAD) COMPUTED By APPLYING (21)

\begin{tabular}{|c|c|c|c|c|c|c|}
\hline $\begin{array}{l}\Delta \boldsymbol{\varphi}_{\mathrm{s},} \\
\Delta \boldsymbol{\varphi}_{\mathrm{VT}}\end{array}$ & $\mathbf{0 . 1 5}$ & $\mathbf{0 . 3}$ & $\mathbf{0 . 6}$ & $\mathbf{0 . 9}$ & $\mathbf{1 . 2}$ & $\mathbf{1 . 8}$ \\
\hline $\mathbf{0 . 1 5}$ & 0.26 & 0.39 & 0.66 & 0.93 & 1.19 & 1.73 \\
\hline $\mathbf{0 . 3}$ & 0.38 & 0.51 & 0.77 & 1.03 & 1.29 & 1.81 \\
\hline $\mathbf{0 . 6}$ & 0.62 & 0.75 & 0.99 & 1.24 & 1.48 & 1.97 \\
\hline $\mathbf{0 . 9}$ & 0.88 & 1.00 & 1.23 & 1.46 & 1.69 & 2.15 \\
\hline $\mathbf{1 . 2}$ & 1.15 & 1.26 & 1.47 & 1.69 & 1.90 & 2.33 \\
\hline $\mathbf{1 . 8}$ & 1.72 & 1.81 & 2.00 & 2.18 & 2.36 & 2.73 \\
\hline
\end{tabular}

obtained by applying the reference method (MCM, Table IV), formula (17) (Table V) and approximation (21) (Table VI). As expected, there are no difference, except very small one due to the limited number of Monte Carlo iterations, between the results in Tables IV and V. From Table VI, it can be noted that even the approximated formula (21) works fine. The maximum error is 0.08 crad, which can be considered acceptable by considering that it affects limits of the confidence interval that are in the order of many crad.

Hence, the proposed simplified expressions allow the operator to estimate at a glance the uncertainties affecting amplitude and phase measurements performed by the typical system shown in Fig. 1. Only the accuracy classes of both the devices must be known.

\section{B. Powermeter}

As in the previous example, the results of an MCM with 100000 iterations have been taken as reference and compared with the results provided by the combined application of (34) and (35).

Different combinations of LPITs and Powermeter accuracies have been studied. As for the LPITs, it has been chosen to consider LPVT and LPCT (along with their relevant Converters) as belonging to same accuracy class. According to Table VII, which is based on [2] and [3], this means that they have the same ratio error but may feature different phase errors. Accuracy classes up to 0.5 have been taken into account.

As for the Powermeter, gain error equal to $0.2 \%$ and $0.5 \%$ have been considered, thus leading to a total number of 12 different case studies. Last, always in the light of referring to practical application, all the previous combinations have been tested with power factor $\cos \varphi$ of 0.8 and 0.5 .

Fig. 6 is the plot of the histogram of $(\Delta P /(P))$, achieved from the MCM when the accuracy class of the LPITs is 0.1, $g=0.2 \%$ and $\cos \varphi=0.8$, along with the Normal pdf having the same mean value and variance as those provided by the 


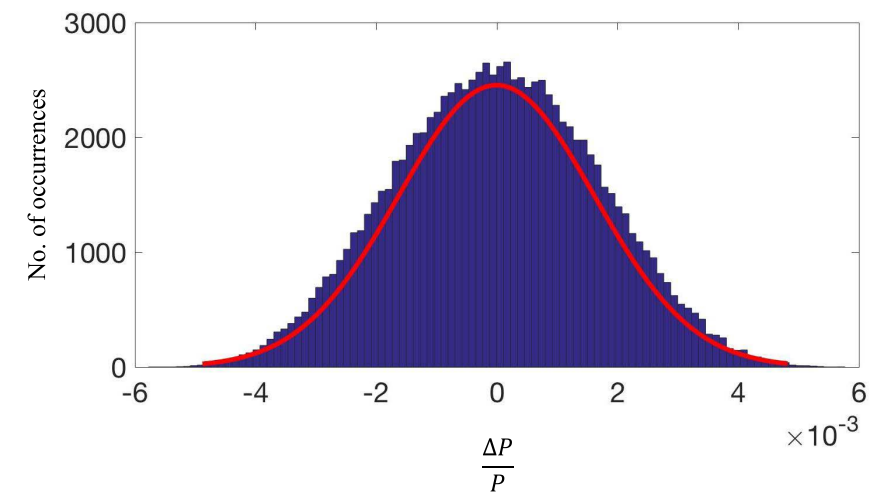

Fig. 6. Estimated pdf achieved from the MCM and best fitting Normal distribution.

TABLE VII

RATIO ERRORS AND PHASE ERRORS FOR LPCT AND LPVT

\begin{tabular}{|c|c|c|c|}
\hline$\varepsilon_{\mathrm{CT}}(\mathbf{\%})$ & $\varepsilon_{\mathrm{VT}} \mathbf{( \% )}$ & $\Delta \varphi_{\mathrm{CT}}$ (crad) & $\Delta \varphi_{\mathrm{VT}}$ (crad) \\
\hline 0.1 & 0.1 & 0.15 & 0.15 \\
\hline 0.2 & 0.2 & 0.3 & 0.3 \\
\hline 0.5 & 0.5 & 0.9 & 0.6 \\
\hline
\end{tabular}

TABLE VIII

REFERENCE $U_{95}$, REF AND ESTIMATED $U_{95, \text { EST }}$ COVERAGE INTERVAL AND CORRESPONDING DIFFERENCE $\Delta U_{95}$ FOR VARIOUS ACCURACY Class and POWER FaCtor 0.8. ACCURACY Class IS THE SAME FOR LPVT AND LPCT

\begin{tabular}{|c|c|c|c|}
\hline & \multicolumn{3}{|c|}{$g=0.2 \%$} \\
\hline Accuracy Class & $U_{95, R E F}(\%)$ & $U_{95, E S T}(\%)$ & $\Delta U_{95}(\%)$ \\
\hline 0.1 & \pm 0.32 & \pm 0.34 & 0.02 \\
\hline 0.2 & \pm 0.53 & \pm 0.54 & 0.01 \\
\hline 0.5 & \pm 1.22 & \pm 1.26 & 0.04 \\
\hline
\end{tabular}

TABLE IX

Reference $U_{95}$, REF AND Estimated $U_{95}$,est Coverage Interval and CORRESPONDING DIFFERENCE $\Delta U_{95}$ FOR VARIOUS ACCURACY Class and POWER FACTOR 0.8. ACCURACY Class IS THE SAME FOR LPVT AND LPCT

\begin{tabular}{|c|c|c|c|}
\hline & \multicolumn{3}{|c|}{$g=0.5 \%$} \\
\hline Accuracy Class & $U_{95, R E F}(\%)$ & $U_{95, E S T}(\%)$ & $\Delta U_{95}(\%)$ \\
\hline 0.1 & \pm 0.56 & \pm 0.63 & 0.07 \\
\hline 0.2 & \pm 0.72 & \pm 0.76 & 0.04 \\
\hline 0.5 & \pm 1.29 & \pm 1.37 & 0.08 \\
\hline
\end{tabular}

MCM. Even if it looks a very good fitting, the statistical tests, as said above, do not support such a conclusion.

Tables VIII and IX show, for $\cos \varphi=0.8, g=0.2 \%$, and $g=0.5 \%$, respectively, the $95 \%$-coverage interval $U_{95, \mathrm{REF}}$ of $(\Delta P /(P))$ obtained from the MCM, the 95\%-coverage interval $U_{95}$, EST of $(\Delta P /(P))$ obtained from (34) and (35) by taking $k=2$, and their difference $\Delta U_{95}$. This last quantity represents, in turns, the error of the proposed simplified expression.

Tables $\mathrm{X}$ and XI show the same above described quantities but in the case of $\cos \varphi=0.5$.

The main conclusions that can be drawn from the above Tables are that the combined application of (34) and (35) provides a very good approximation of the correct value of the
TABLE $X$

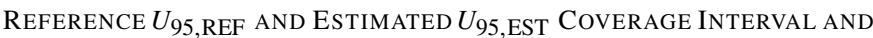
CORRESPONDING DIFFERENCE $\Delta U 95$ FOR VARIOUS ACCURACY Class and Power Factor 0.5. ACCURACy Class IS THE SAME FOR LPVT AND LPCT

\begin{tabular}{|c|c|c|c|}
\hline & \multicolumn{3}{|c|}{$g=0.2 \%$} \\
\hline Accuracy Class & $U_{95, R E F}(\%)$ & $U_{95, E S T}(\%)$ & $\Delta U_{95}(\%)$ \\
\hline 0.1 & \pm 0.50 & \pm 0.51 & 0.01 \\
\hline 0.2 & \pm 0.92 & \pm 0.94 & 0.02 \\
\hline 0.5 & \pm 2.21 & \pm 2.32 & 0.11 \\
\hline
\end{tabular}

TABLE XI

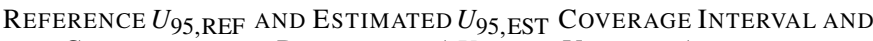
CORRESPONDING DIFFERENCE $\Delta U_{95}$ FOR VARIOUS ACCURACY Class and Power FaCtor 0.5. ACCURACY Class IS THE SAME FOR LPVT AND LPCT

\begin{tabular}{|c|c|c|c|}
\hline & \multicolumn{3}{|c|}{$g=0.5 \%$} \\
\hline Accuracy Class & $U_{95, R E F}(\%)$ & $U_{95, E S T}(\%)$ & $\Delta U_{95}(\%)$ \\
\hline 0.1 & \pm 0.69 & \pm 0.73 & 0.04 \\
\hline 0.2 & \pm 1.06 & \pm 1.08 & 0.02 \\
\hline 0.5 & \pm 2.29 & \pm 2.38 & 0.09 \\
\hline
\end{tabular}

95\%-confidence interval. In fact, the maximum $\Delta U_{95}$ value, which represents the error of the proposed approach, is about $0.1 \%$ which is more or less $1 / 20$ of corresponding $U_{95, \text { REF }}$. Moreover, when the considered measurement system exhibits its lower uncertainty (approximately $0.3 \%$ ), $\Delta U_{95}$ still remains in the same relationship with $U_{95, \mathrm{REF}}$, taking a value of about $0.02 \%$.

\section{CONClusion}

In this paper, expressions for evaluating uncertainty in measurements performed by modern measurement systems have been presented. Authors have focused their study in obtaining uncertainty expressions usable by system and industry operators. The aim of this work has been to take first into account the numerous requests coming from the world of system users to have available ready-to-use expressions for evaluating at a glance the uncertainty affecting the most important parameters measured by IEDs (phasors of voltage and currents and powers at rated frequency). In the present work, performed specifically for this purpose, authors have come-up with simplified expressions of uncertainty. Numerical validation, aimed at verifying their effectiveness and robustness with different combinations of uncertainty values of each measurement item, have shown that the difference between the results of uncertainties evaluated by such expressions and those obtained by using MCM, assumed as reference, are far below the evaluated uncertainties. This way it can be concluded that the aforementioned expressions can be effectively taken into considerations for being applied in practical applications. In this regard, the coming Standard IEC 61869-13, relevant to the definition and specification of the SAMU, suggest to use the method proposed in [10] as one method for evaluating uncertainty through the two items made by LPIT and SAMU.

\section{REFERENCES}

[1] Instrument Transformers-Part 6: Additional General Requirements for Low-Power Instrument Transformers, document IEC 61869-6:2016, International Standardization Organization, Geneva, Switzerland, 2016. 
[2] Instrument Transformers-Part 10: Additional Requirements for Low Power Stand Alone Current Sensors, document IEC 61869-10, International Standardization Organization, Geneva, Switzerland, 2018.

[3] Instrument Transformers-Part 11: Additional Requirements for Low Power Stand Alone Voltage Sensors, document IEC 61869-11, International Standardization Organization, Geneva, Switzerland, 2018.

[4] Instrument Transformers-Part 13: Stand Alone Merging Unit, document IEC 61869-13, International Standardization Organization, Geneva, Switzerland, 2018

[5] Communication Networks and Systems for Power Utility AutomationPart 9-2: Specific Communication Service Mapping (SCSM)Sampled Values Over ISO/IEC 8802-3, document IEC 61850-9-2:2011, International Standardization Organization, Geneva, Switzerland, 2008.

[6] Uncertainty of Measurement-Part 3: Guide to the Expression of Uncertainty in Measurement (GUM:1995), document ISO/IEC Guide 98-3:2008, International Standardization Organization, Geneva, Switzerland, 2008.

[7] Evaluation of Measurement Data-Supplement 1 to the 'Guide to the Expression of Uncertainty in Measurement'-Propagation of Distributions Using a Monte Carlo Method, document ISO/IEC Guide 98-3/Suppl.1:2008, International Standardization Organization, Geneva, Switzerland 2008.

[8] A. Benigni, F. Ponci, and A. Monti, "Towards an uncertainty-based model level selection for the simulation of complex power systems," in Proc. Complex. Eng. (COMPENG), 2010, pp. 46-48.

[9] H. Choi, P. J. Seiler, and S. V. Dhople, "Propagating uncertainty in power-system DAE models with semidefinite programming," IEEE Trans. Power Syst., to be published.

[10] L. Peretto, R. Tinarelli, and K. Yiğit, "Uncertainty evaluation in measurement equipments for power systems," in Proc. IEEE AMPS, Aachen, Germany, Sep. 2016, pp. 1-5.

[11] G. Mauris, V. Lasserre, and L. Foulloy, "A fuzzy approach for the expression of uncertainty in measurement," Measurement, vol. 29, no. 3 , pp. 165-177, 2001.

[12] M. K. Urbanski and J. Wạsowski, "Fuzzy approach to the theory of measurement inexactness," Measurement, vol. 34, no. 1, pp. 67-74, 2003.

[13] S. Salicone, Measurement Uncertainty-An Approach via the Mathematical Theory of Evidence. New York, NY, USA: Springer, 2007.

[14] P. da Silva Hack and C. S. ten Caten, "Measurement uncertainty: Literature review and research trends," IEEE Trans. Instrum. Meas., vol. 61 , no. 8, pp. 2116-2124, Aug. 2012.

[15] A. Papoulis, Probability, Random Variables and Stochastic Processes, 3rd ed. New York, NY, USA: McGraw-Hill, 1991.

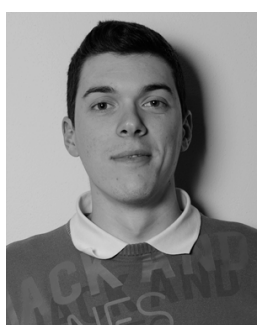

Alessandro Mingotti (S'17) received the M.S. degree in electrical engineering from the University of Bologna, Bologna, Italy, in 2016, where he is currently pursuing the Ph.D. degree in biomedical, electrical, and systems engineering.

His current research interests include measurements for smart grids and electrical safety.

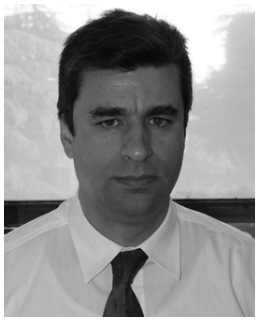

Lorenzo Peretto (M'98-SM'03) is currently a Professor of Electrical and Electronic Measurements with the University of Bologna, Bologna, Italy. He is also a Consultant of industries operating in the field of instrumentation and sensors for electrical measurements. He has authored or co-authored more than 200 papers and 3 books, and holds 24 patents. His current research interests include the design and calibration of voltage and current instrument transformers for medium and high-voltage power networks, the design and realization of calibration systems of voltage and current instrument transformers, and the measurements of electrical quantities in power networks.

Dr. Peretto is a member of the IEEE Instrumentation and Measurement Society. He is the Chairman of the Annual IEEE Applied Measurements for Power System Conference, a member of the IEC TC38 "Instrument Transformers," and the Chairman of the TC38/WG45 "Standard Mathematical Models for Instrument Transformers" and of the TC38/WG53 "Uncertainty Evaluation in the Calibration of Instrument Transformers."

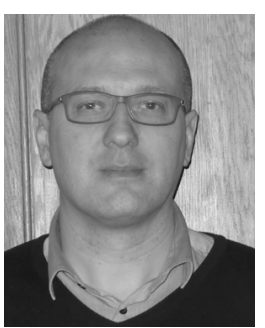

Roberto Tinarelli (S'02-M'05-SM'14) received the M.S. degree in electrical engineering from the University of Bologna, Bologna, Italy, in 2000, and the Ph.D. degree in electrical engineering from the Polytechnic of Milan, Milan, Italy, in 2004.

$\mathrm{He}$ is currently an Associate Professor of Electrical Measurements with the Department "Guglielmo Marconi" of the University of Bologna. He has authored or co-authored more than 120 scientific papers and 1 book and the Co-Inventor of some WO patents in the field of sensors and instrument tranformers. His current research interests include the design and metrological characterization of instruments for measurement under nonsinusoidal conditions, the design and development of new instrumentation for flicker measurement, the design and characterization of new electromagnetic-fields sensors and instrumentation, and the reliabiltity study of electronic devices.

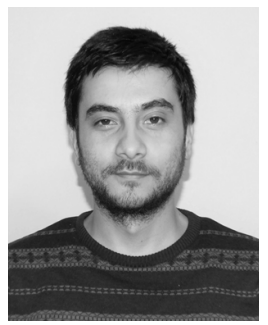

Kenan Yiğit received the M.Sc. degree in electrical engineering from Yildiz Technical University, Istanbul, Turkey, in 2013, where he is currently pursuing the Ph.D. degree in electrical engineering.

In 2013, he joined the Marine Engineering Operation Department, Yildiz Technical University, where he is currently a Research Assistant. In 2016, he joined the Electrical and Electronic Measurement Group, Department of Electrical Engineering, University of Bologna, Bologna, Italy. His current research interests include the measurement of electric power systems, energy management, smart grid, and ship electrical system. 